\title{
Expression Recognition in Videos Using a Weighted Component-Based Feature Descriptor
}

\author{
Xiaohua Huang ${ }^{1,2}$, Guoying Zhao ${ }^{1}$, Matti Pietikäinen ${ }^{1}$, and Wenming Zheng ${ }^{2}$ \\ 1 Machine Vision Group, Department of Electrical and Information Engineering, \\ University of Oulu, Finland \\ 2 Research Center for Learning Science, Southeast University, China \\ \{huang.xiaohua, gyzhao,mkp\}@ee.oulu.fi, \\ wenming_zhengaseu.edu.cn \\ http://www.ee.oulu.fi/mvg
}

\begin{abstract}
In this paper, we propose a weighted component-based feature descriptor for expression recognition in video sequences. Firstly, we extract the texture features and structural shape features in three facial regions: mouth, cheeks and eyes of each face image. Then, we combine these extracted feature sets using confidence level strategy. Noting that for different facial components, the contributions to the expression recognition are different, we propose a method for automatically learning different weights to components via the multiple kernel learning. Experimental results on the Extended Cohn-Kanade database show that our approach combining component-based spatiotemporal features descriptor and weight learning strategy achieves better recognition performance than the state of the art methods.
\end{abstract}

Keywords: Spatiotemporal features, LBP-TOP, EdgeMap, Information fusion, Multiple kernel learning, Facial expression recognition.

\section{Introduction}

A goal of automatic facial expression analysis is to determine the emotional state, e.g., happiness, sadness, surprise, neutral, anger, fear, and disgust, of human beings based on facial images, regardless of the identity of the face. To date, there have been some surveys describing the state-of-the-art techniques of facial expression recognition, based on static images or video sequences [423]. The surveys show that dynamic features from video sequences can provide more accurate and robust information than the static features from images.

Feature representation is very important for automatic facial expression analysis. Methods combining geometric and appearance features have been considered earlier [23]. For example, Tian et.al [22] proposed to use facial component shapes and transient features like crow-feet wrinkles and nasal-labial furrows. A framework of combining facial appearance (Scale-invariant feature transform) and shape information (Pyramid histogram of orientated gradient) was proposed for facial expression recognition [15]. Both similarity-normalized shape (SPTS) and canonical appearance (CAPP) were derived from the active appearance models (AAM) to interpret the face images [16]. It is 
observed that the combination of geometric and appearance information can describe the face in a better way. But most of approaches used complicated geometric information like shapes and the trasient feature [1622]. Furthermore some researches [8 8 10 $15 \mid 20]$ cropped the facial image into some sub-regions or components, and then extracted the appearance features from those components. Researches [810] have shown that component-based approach is robust in some cases against pose motion or partial occlusion. But the features in the earlier component-based methods were only extracted from static images, even though some of them [2024] use Hidden Markov Models (HMMs) or Dynamic Bayesian Networks (DBNs) to integrate the static information with time development.

In facial expression recognition, it is notable that the facial components take distinct effect on different expressions [13 1921]. Hence, another major task in this paper is to select the most relevant features among the multiple feature sets extracted from the different facial regions. Using boosting algorithm or just simply assign the weight parameters to the corresponding face components [21,28] would be feasible. Recently, the multiple kernels learning (MKL) in support vector machines (SVM) has been introduced to combine heterogeneous sources of information for decision fusion in computer vision. In addition, recent studies in [37] have shown that the MKL method achieves decent performance in applications of object/image recognition.

In this paper, we focus on combining dynamic texture features and structural features of facial components for describing facial movement. In order to reduce the complexity, we develop a method for fusing multiple feature representations. Motivated by the aforementioned MKL method, we aim to learn weights for multiple feature sets in facial components. We test our approache on the Extended Cohn-Kanade Database which contains 97 subjects with seven emotions. Our person-independent experiment shows that the weighted component-based approach performs better than other approaches. The contributions of this paper include: 1) we applied the dynamic texture and structural shape features [5|27] from facial components to represent facial dynamic sequences; 2) we developed the framework of feature fusion via confidence level method, and 3) weight learning by MKL was presented.

\section{Component-Based Feature Descriptor}

Concerning pose variation and partial occlusion, component-based features [8 10 [15] are more effective for representing facial expressions. Three facial components: mouth, cheeks and eyes are considered in our method and shown in Figure 1(c). Facial points, shown in Figure 1 a) in each frame can be obtained by AAM [2]. Then eyes, nose and mouth areas are determined by the detected 62 facial points. Please note that because eyebrows are also important in expression, the eyes component is extended from the blue dashed rectangle to blue solid rectangle, as shown in Figure 1 b). In order to extract micro-information from components, each component is further divided into a couple of blocks, as shown in Figure 2 b). In this paper, we use CFD ( $\underline{C}$ omponent-based $\underline{F}$ eature $\underline{D}$ escriptor) as the abbreviation of the proposed framework. 


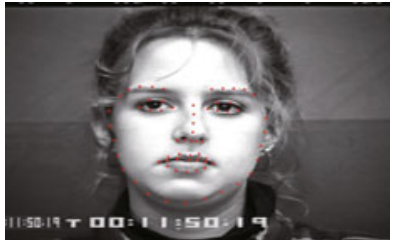

(a)

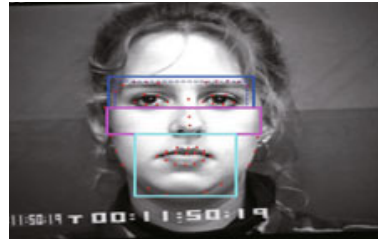

(b)

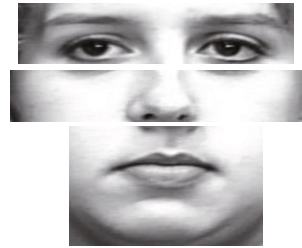

(c)

Fig. 1. (a) 62 facial points detected by AAM [2]16] (b) Rectangles for eyes, nose, mouth determined by detected facial points (c) Three components cropped from the facial image-eyes, nose and mouth

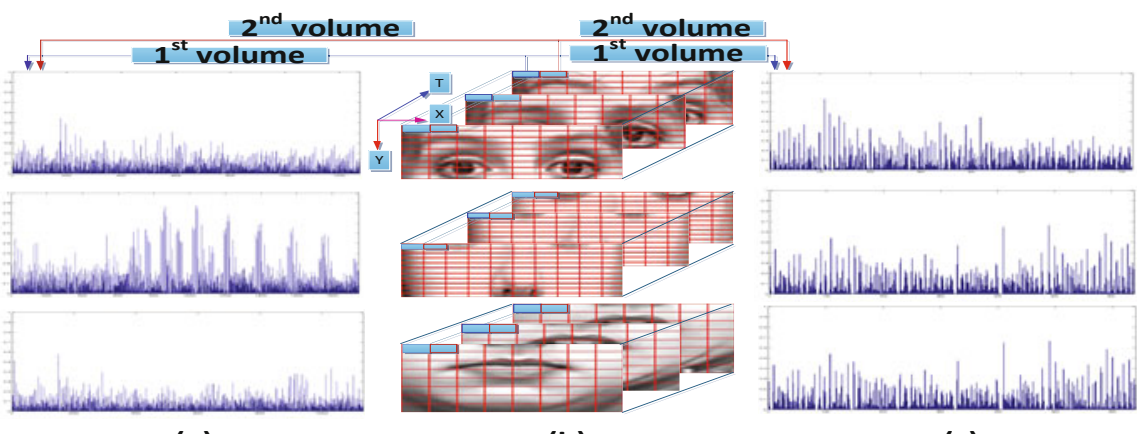

(a)

(b)

(c)

Fig. 2. Framework of component-based feature descriptor. (a) Dynamic appearance representation by LBP-TOP; (b) Three components (eyes, nose, mouth); (c) Dynamic shape representation by EdgeMap.

\subsection{Dynamic Texture Features For Appearance Representation}

The local binary pattern (LBP) operator is a gray-scale invariant texture primitive statistic, which has shown excellent performance in the classification of various kinds of textures [18]. And LBP operator is defined as:

$$
L B P_{S, R}=\sum_{s=0}^{S-1} f\left(g_{s}-g_{c}\right) 2^{s},
$$

where

$$
f\left(g_{s}-g_{c}\right)=\left\{\begin{array}{l}
1, g_{s}-g_{c} \geq 0 \\
0, g_{s}-g_{c}<0
\end{array},\right.
$$

and $g_{c}$ is the gray value of the center pixel, $g_{s}$ is the gray values of $S$ equally spaced pixels on a circle of radius $R$ at this center pixel.

LBP-TOP has been recently proposed for motion analysis and shown excellent performance in the classification of expression [28] and lip-reading [25]. Features extracted by this method effectively describe the appearance, horizontal motion and vertical motion from the image sequence. We extend to use LBP-TOP to describe the spatiotemporal features (XY, XT, and YT planes) of three components. That is to say, after detecting 
each component, the LBP-TOP histograms for each component are computed and concatenated into a single histogram to represent the appearance (XY plane) and motion (XT and YT planes) of the facial expression sequence, shown in Figure 2(a). In our experiments, the radii in axes $X, Y$ and $T$ are set as three; the number of local neighboring points around the central pixel for all three planes is set as eight.

\subsection{Dynamic Structural Features For Shape Representation}

Edge map (EdgeMap) features were recently proposed to describe the edge orientation of the pixel for detecting facial landmarks [5] and also utilized as describing structural features together with LBP (only in XT and YT planes) for speaker identification from lipreading [26]. Given a smoothed image from one video with a set of 16 kernels, the whole set of 16 kernels results from the differences between two oriented Gaussians with shifted kernel:

$$
G_{\theta_{t}}=\frac{G_{\theta_{t}}^{-}-G_{\theta_{t}}^{+}}{\sum_{u, v}\left[\left(G_{\theta_{t}}^{-}-G_{\theta_{t}}^{+}\right) \cdot h\left(G_{\theta_{t}}^{-}-G_{\theta_{t}}^{+}\right)\right]},
$$

where

$$
\begin{aligned}
G_{\theta_{t}}^{-}= & \frac{1}{2 \pi \sigma^{2}} \exp \left(-\frac{\left(u-\sigma \cos \theta_{t}\right)^{2}+\left(v-\sigma \sin \theta_{t}\right)^{2}}{2 \sigma^{2}}\right), \\
G_{\theta_{t}}^{+}= & \frac{1}{2 \pi \sigma^{2}} \exp \left(-\frac{\left(u+\sigma \cos \theta_{t}\right)^{2}+\left(v+\sigma \sin \theta_{t}\right)^{2}}{2 \sigma^{2}}\right), \\
& h\left(G_{\theta_{t}}^{-}-G_{\theta_{t}}^{+}\right)=\left\{\begin{array}{l}
1, G_{\theta_{t}}^{-}-G_{\theta_{t}}^{+}>0 \\
0, G_{\theta_{t}}^{-}-G_{\theta_{t}}^{+} \leqslant 0
\end{array}\right.
\end{aligned}
$$

and $\sigma$ is a root mean square deviation of the Gaussian distribution, $\theta_{t}$ is angle of the Gaussian rotation, $\theta_{t}=22.5 \times t, t=0, \cdots, 15 ; u, v=-3,-2,-1,0,1,2,3$.

In our paper, same to [5], 10 kernels $(t=2,3,4,5,6,10,11,12,13,14)$ is used to define the contrast magnitude of a local edge at pixel $(p, q)$. The orientation of a kernel that gave the maximum response is estimated by the orientation of a local edge:

$$
\vartheta_{p, q, \theta_{t}}=\sum_{c, d} g_{p-u, q-v} G_{\theta_{t}}
$$

where $g_{p, q}$ denotes the gray level of the image at pixel $(p, q) ; p=0, \cdots, W-1$, $q=0, \cdots, H-1 ; W$ and $H$ are the width and height of the image, respectively.

After getting the edge orientation for each pixel, a histogram is created to collect up the occurrences of different orientations. Studies in [626] used EdgeMap to describe the structural features in XY plane of a video sequence. Inspired by them, the EdgeMap histograms from XY plane in block volumes (Figure 2 b)) are concatenated into a single histogram for representing structural features, shown in Figure 2(c). But different from [26], in which they only considered LBP texture features in XT and YT planes, in our approach, LBP operation in XY plane is still utilized to describe appearance features and then concatenated into a single histogram together with those from XT and YT planes, shown in Figure 2]a), in order to avoid the loss of appearance feature. 


\section{Multiple Feature Fusion}

As shown in Figure 2, we extract component-based feature sets, which represents texture features from LBP-TOP operation or structural features from EdgeMap in each component. In studies on various feature extraction approaches, it has been suggested that different feature sets could offer complementary information. A fusion scheme that harnesses various representations is likely to improve the overall performance. The outputs of various feature extractors can be fused to obtain decisions that are more accurate than the decisions made by any individual feature representation. As shown in Figure 3 , each feature set of LBP-TOP or EdgeMap is the input of one matching module and the model of weight learning.

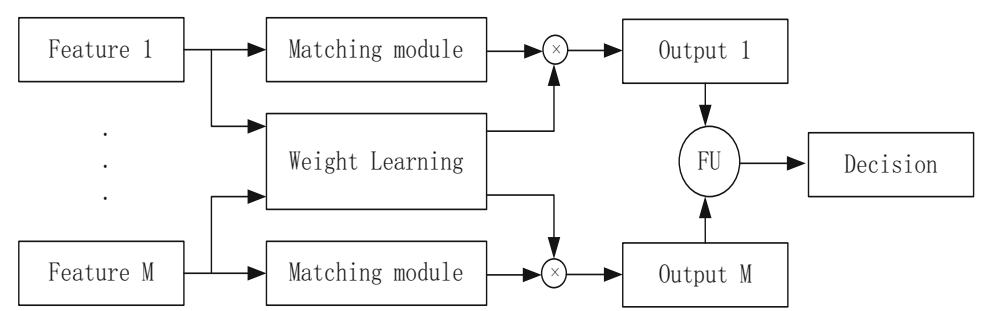

Fig. 3. Framework of multiple feature fusion, FU: Fusion Module

\subsection{The Weight Learning Framework}

The MKL approach [3714] is used to combine or select relevant representations. The multiple kernel learning tasks in our approach are viewed as an efficient way to learn weights that are optimal for several feature sets. Suppose we have $M$ feature sets $\left\{\boldsymbol{\Omega}_{m}\right\}_{m=1}^{M}$, each of them has $N$ samples $\left\{\vec{x}_{i, m}\right\}_{i=1}^{N}$, and the corresponding class label of $\vec{x}_{i, m}$ is $y_{i}$, where $y_{i} \in\{+1,-1\}$. Thus, the kernel function of MKL is defined as

$$
k_{i, j}=\sum_{m=1}^{M} \beta_{m} k\left(\vec{x}_{i, m}, \vec{x}_{j, m}\right),
$$

s.t. $\beta_{m} \geq 0$ and $\sum_{m=1}^{M} \beta_{m}=1$, where $\beta_{m}$ is the weight of $m$-th feature set $\boldsymbol{\Omega}_{m}$, $k\left(\vec{x}_{i, m}, \vec{x}_{j, m}\right)$ is the base kernel of $\vec{x}_{i, m}$ and $\vec{x}_{j, m}$ from $\boldsymbol{\Omega}_{m}$. Here, all kernel matrices of feature sets have been normalized to unit trace.

The MKL task, which is based on the framework of SVM, is considered as a way of optimizing the kernel weights. When a kernel machine for multiple feature sets is used, the dual problem of MKL is defined as

$$
\max \sum_{i=1}^{N} \alpha_{i}-\frac{1}{2} \sum_{i=1}^{N} \sum_{j=1}^{N} \alpha_{i} \alpha_{j} y_{i} y_{j} \sum_{m=1}^{M} \beta_{m} k\left(\vec{x}_{i, m}, \vec{x}_{j, m}\right),
$$

s.t. $\sum_{i=1}^{N} y_{i} \alpha_{i}=0,0 \leq \alpha_{i} \leq \varepsilon$ and $\beta_{m} \geq 0, \sum_{m=1}^{M} \beta_{m}=1$, where, $\alpha_{i}$ is the Lagrange coefficient, and the regularization $\varepsilon$ determines the trade-off between the margin and the error on training data. 
Optimizing over both the Lagrange coefficient $\alpha_{i}$ and the weights for $m$-th feature set $\beta_{m}$ is one particular form of semidefinite programming (SDP). MKL algorithm is terminated when a stopping criterion is met. The stop criterion in our implementation is based on the variation of coefficients $\beta_{m}$ between two consecutive steps. In order to compute the weights for different feature sets from specific expression, we should consider an approach that divides multi-class problem into some one-vs-rest classification problems. Finally, this framework will generate the weight of $m$-th feature set under $c$-th class, i.e. $\beta_{c, m}$. In our case, we use MKL-WL (MKL for Weight Learning) for abbreviation, which is summarized in Algorithm 1

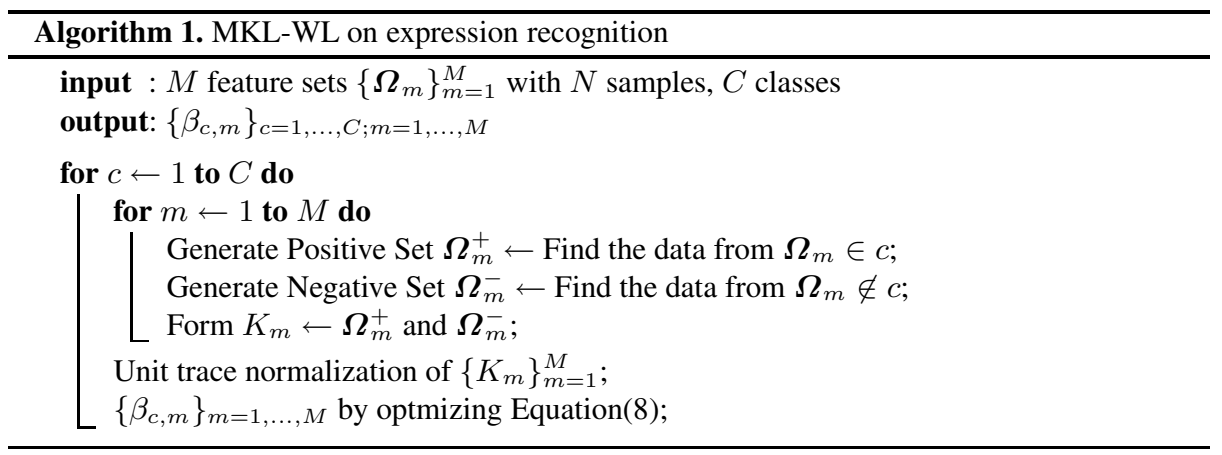

\subsection{The Fusion Module Framework}

For exploiting the complementary information among all feature sets, we investigated one decision rule, i.e. mean rule. Detailed derivation of decision rules can be found e.g. in [12]. Assume that all feature sets are generally statistically independent, and the priori probability of occurrence for $c$-th class model are under assumption of equal priors, the fusion rule of multiple feature sets [9] is described as:

Assign $\vec{x} \rightarrow \mu$ if

$$
\begin{aligned}
P\left(c=\mu \mid \overrightarrow{\boldsymbol{x}}, \boldsymbol{\Omega}_{1}, \ldots, \boldsymbol{\Omega}_{M}\right) & =\max _{c \in\{1, \ldots, C\}}\left[\boldsymbol{Q}\left(\left\{P\left(c \mid \boldsymbol{\Omega}_{1}\right) \beta_{c, 1}, \ldots, P\left(c \mid \boldsymbol{\Omega}_{M}\right) \beta_{c, M}\right\}\right)\right] \\
& =\max _{c \in\{1, \ldots, C\}} \frac{\sum\left\{P\left(c \mid \boldsymbol{\Omega}_{1}\right) \beta_{c, 1}, \ldots, P\left(c \mid \boldsymbol{\Omega}_{M}\right) \beta_{c, M}\right\}}{M}(9)
\end{aligned}
$$

where $\vec{x}$ and $\mu$ represents the testing sample and the corresponding class, respectively.

In our framework, LIBSVM [1] is used for modeling matching module and generating voting numbers or probabilities.

\section{Experiments}

The proposed approach was evaluated on the Extended Cohn-Kanade facial expression database $(\mathrm{CK}+)$ [16]. The orginal Cohn-Kanade database [11] includes 486 FACScoded sequences from 97 subjects for six basic expressions: happiness, sadness, surprise, anger, disgust and fear. For $\mathrm{CK}+$ distribution, it has been further augmented to include 593 sequences from 123 subjects for seven expressions (additional 107 sequences, 


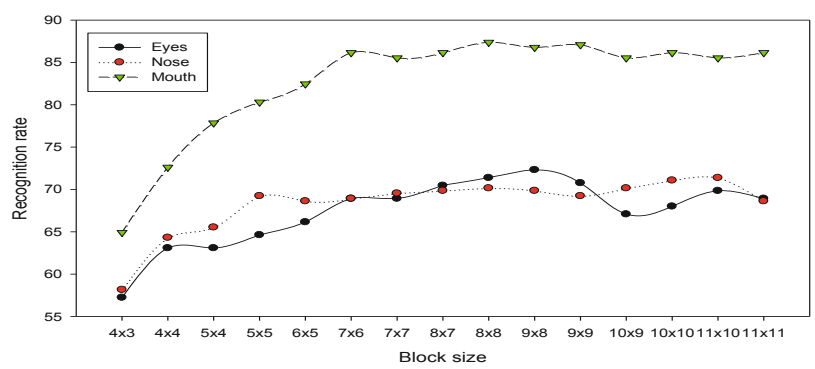

Fig. 4. Performance of three components with different block sizes

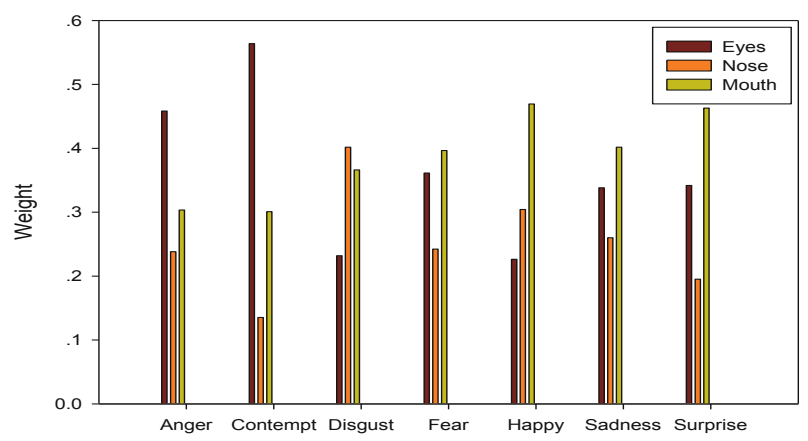

Fig. 5. Average weights for three components based on three kernels

26 subjects and contempt expression), which makes it more challenging than the original database. In our experiments, 325 sequences from 118 subjects were selected from the database for seven basic expression recognition. Leave-one-subject-out method was used in the whole scenario.

In our approach, three components are cropped as shown in Figure 1. However, the size of each facial component is so large that more than one block is needed to describe its local spatiotemporal information. Moreover, as observed from Figure 1(c), the areas of different parts are different. This means that using the same number of blocks for all components is not reasonable [27]. Thus, different number of blocks is used for three components. Figure 4 shows the performance of using different block numbers of eyes, nose, and mouth. As observed from Figure 4 , the highest recognition rate is achieved when the block size is $9 \times 8,11 \times 10,8 \times 8$, for eyes, nose and mouth, respectively. Based on the results in Figure $49 \times 8,11 \times 10,8 \times 8$ block sizes are used in eyes, nose and mouth, respectively.

In Section 3.1, we proposed dynamic weight learning by using MKL. In order to figure out the importance of the components to different expressions, the weights on three components are shown in Figure 5. In this figure, these weights clearly show: (1) both eyes and mouth components play important roles in fear and sadness; (2) both mouth and nose components contribute to disgust and happiness; (3) anger and contempt, mostly depend on eyes; (4) mouth component determines surprise. 
Below, we give brief comparison with the state-of-the-art works [1627] for expression recognition. Figure 6 compares our methods: CFD, CFD with $\underline{M}$ KL based on linear kernel (CFDM-Linear), $\underline{H}$ istogram $\underline{I}$ ntersection kernel [17] (CFDM-HI), and Gaussian kernel (CFDM-Gaussian), with SPTS+CAPP [16], LBP-TOP [27] and EdgeMap [6]. From this figure, we can see that CFD obtained better result $(89.85 \%)$ than block-based LBP-TOP (87.07\%) and EdgeMap (82.77\%), with increase of $2.77 \%$ and $7.08 \%$, and also better than SPTS+CAPP (88.38\%), with an increase $1.47 \%$. Additionally, it is very interesting that dynamic weight using MKL based on linear kernel $(93.23 \%)$ and HI kernel $(93.85 \%)$ can improve the performance of CFD. Compared to the other methods, CFDM-HI outperformed on average recognition rate and all expressions except anger.

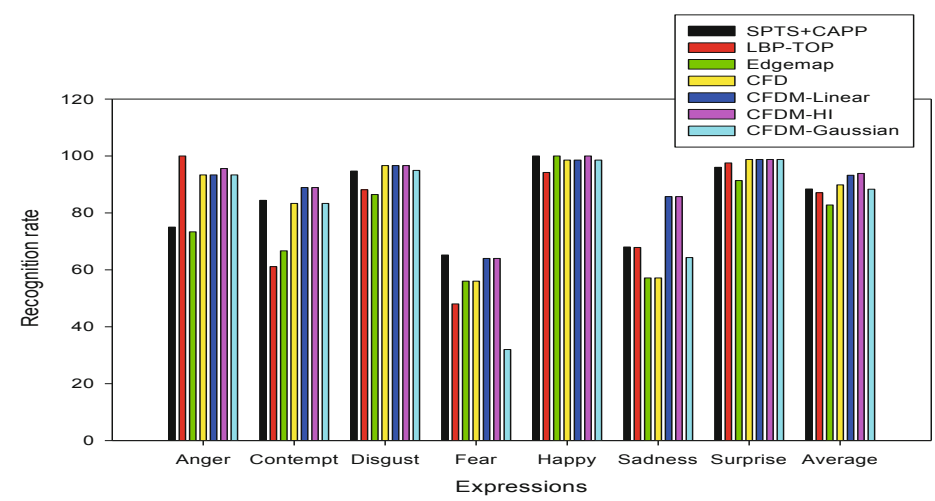

Fig. 6. Performance comparison (\%) with different approaches

\section{Conclusion}

In order to boost facial expression recognition, we propose a component-based feature descriptor to describe facial expressions from video sequences. In our approach, three components (eyes, nose and mouth) are cropped from facial image according to automatically detected facial points, and dynamic texture and shape features are extracted by LBP-TOP and EdgeMap, respectively. Then multiple feature sets are combined by fusion strategy. Furthermore, for boosting CFD, we proposed an approach for learning weights for multiple feature sets.

In experiments on the $\mathrm{CK}+$ Database, we discussed the roles and significance of components with respect to expressions, through analyzing the weights computed by MKLWL algorithm. Besides, we also have demonstrated that the CFDM-Linear, CFDM-HI lead to a promising improvement in facial expression classification, comparing with previous works. In future work we plan to explore how our approach could be adopted to the very challenging problems including view variation and partial occlusion.

\section{Acknowledgement}

The financial support provided by the Academy of Finland is gratefully acknowledged. The first author is funded by China Scholarship Council of Chinese government. This 
work was partly supported by Natural Science Foundations of China under Grant 61073137, and partly by the Jiangsu Natural Science Foundations under Grant BK2010243. The authors would like to thank the anonymous reviewers for their constructive advice.

\section{References}

1. Chang, C., Lin, C.: Libsvm: a library for support vector machines, Software available as http://www.csie.ntu.edu/tw/ cjlin/libsvm

2. Cootes, T., Edwards, G., Taylor, C.: Active appearance models. IEEE Transactions on Pattern Analysis and Machine Intelligence 23(6), 681-685 (2001)

3. Dileep, A., Sekhar, C.: Representation and feature selection using multiple kernel learning. In: International Joint Conference on Neural Networks, pp. 717-722. IEEE Press, New York (2009)

4. Fasel, B., Luettin, J.: Automatic facial expression analysis: a survey. Pattern Recognition 36, 259-275 (2003)

5. Gizatdinova, Y., Surakka, V.: Feature-based detection of facial landmarks from neutral and expressive facial images. IEEE Transactions on Pattern Analysis and Machine Intelligence 28(1), 135-139 (2006)

6. Gizatdinova, Y., Surakka, V., Zhao, G., Makinen, E., Raisamo, R.: Facial expression classification based on local spatiotemporal edge and texture descriptor. In: 7th International Conference on Methods and Techniques in Behavioral Research Measuring (2010)

7. Goene, M., Alpaydin, E.: Localized multiple kernel machines for image recognition. In: NIPS 2009 Workshop on Understanding Multiple Kernel Learning Methods. MIT Press, Cambridge (2009)

8. Heisele, B., Koshizen, B.: Components for face recognition. In: IEEE International Conference on Automatic Face and Gesture Recognition, pp. 153-158. IEEE Press, New York (2004)

9. Huang, X., Zhao, G., Pietikäinen, M., Zheng, W.: Dynamic facial expression recognition using boosted component-based spatiotemporal features and multi-classifier fusion. In: BlancTalon, J., Bone, D., Philips, W., Popescu, D., Scheunders, P. (eds.) ACIVS 2010, Part II. LNCS, vol. 6475, pp. 312-322. Springer, Heidelberg (2010)

10. Ivanov, Y., Heisele, B., Serre, T.: Using component features for face recognition. In: IEEE International Conference on Automatic Face and Gesture Recognition, pp. 421-426. IEEE Press, New York (2004)

11. Kanade, T., Cohn, J., Tian, Y.: Comprehensive database for facial expression analysis. In: IEEE International Conference on Automatic Face and Gesture Recognition, pp. 46-53. IEEE Press, New York (2000)

12. Kittler, J., Hatef, M., Duin, R., Matas, J.: On combining classifiers. IEEE Transactions on Pattern Analysis and Machine Intelligence 20(3), 226-239 (1998)

13. Kotsia, I., Buciu, I., Pitas, I.: An analysis of facial expression recognition under partial facial image occlusion. Image and Vision Computing 26(7), 1052-1067 (2008)

14. Lanckriet, G., Cristianini, N., Bartlett, P., Ghaoui, L., Jordan, M.: Learning the kernel matrix with semidefinite programming. Journal of Machine Learning Research 5, 27-72 (2004)

15. Li, Z., Imai, J., Kaneko, M.: Facial-component-based bag of words and phog descriptor for facial expression recognition. In: IEEE International Conference on Systems, Man, and Cybernetics, pp. 1353-1358. IEEE Press, New York (2009) 
16. Lucey, P., Cohn, J., Kanade, T., Saragih, J., Ambadar, Z.: The extended cohn-kanade dataset $(\mathrm{ck}+)$ : a complete dataset for action unit and emotion-specified expression. In: IEEE Conference on Computer Vision and Pattern Recognition Workshops, pp. 94-101. IEEE Press, New York (2010)

17. Maji, S., Berg, A., Malik, J.: Classification using intersection kernel support vector machines is efficient. In: IEEE Conference on Computer Vision and Pattern Recognition. IEEE Press, New York (2008)

18. Ojala, T., Pietikäinen, M., Maenpaa, T.: Multiresolution gray-scale and rotation invariant texture classification with local binary patterns. IEEE Transactions on Pattern Analysis and Machine Intelligence 24(7), 971-987 (2002)

19. Pantic, M., Rothkrantz, L.: Expert system for automatic analysis of facial expressions. Image and Vision Computing 18(11), 881-905 (2000)

20. Sun, Y., Yin, L.: Evaluation of spatio-temporal regional features for 3D face analysis. In: IEEE Computer Vision and Pattern Recognition Workshop, pp. 13-19. IEEE Press, New York (2009)

21. Taini, M., Zhao, G., Pietikäinen, M.: Weight-based facial expression recognition from nearinfrared video sequences. In: 16th Scandinavian Conference on Image Analysis, pp. 239248. Springer, Heidelberg (2009)

22. Tian, Y., Kanade, T., Cohn, J.: Facial expression analysis. In: Li, S.Z., Jain, A.K. (eds.) Handbook of Face Recognition, pp. 247-276. Springer, Heidelberg (2005)

23. Zeng, Z., Pantic, M., Roisman, G., Huang, T.: A survey of affective recognition methods: Audio, visual and spontaneous expression. IEEE Transactions on Pattern Analysis and Machine Intelligence 31(1), 39-58 (2009)

24. Zhang, Y., Ji, Q.: Active and dynamic information fusion for facial expression understanding from image sequences. IEEE Transactions on Pattern Analysis and Machine Intelligence 27(5), 699-714 (2005)

25. Zhao, G., Barnard, M., Pietikäinen, M.: Lipreading with local spatiotemporal descriptors. IEEE Transaction on Multimedia 11(7), 1254-1265 (2009)

26. Zhao, G., Huang, X., Gizadinova, Y., Pietikäinen, M.: Combining dynamic texture and structural features for speaker identification. In: ACM Multimedia 2010 Workshop on Multimedia in Forensics, Security and Intelligence. ACM, New York (2010)

27. Zhao, G., Pietikäinen, M.: Dynamic texture recognition using local binary pattern with an application to facial expressions. IEEE Transactions on Pattern Analysis and Machine Intelligence 29(6), 915-928 (2007)

28. Zhao, G., Pietikäinen, M.: Boosted multi-resolution spatio temporal descriptors for facial expression recognition. Pattern Recognition Letters 30, 1117-1127 (2009) 